\title{
Deflación mamaria en mamoplastía de aumento: Reporte de un caso
}

\author{
Guillermo M. Wiegering Cecchi ${ }^{1}$
}

\section{RESUMEN}

Reportamos el caso de una paciente sometida a aumento mamario con implante relleno con suero salino, el cual sufrió deflación debido a la interposición de un anillo fibroso, ocasionado por la cápsula alrededor de la válvula de sellado. (Horiz Med 2015; 15(1): 71-75)

Palabras clave: Aumento Mamario, Deflación Mamaria, Cápsula. (Fuente: DeCS BIREME).

Mammary deflation: by the way of a case

\section{ABSTRACT}

We report an unusual case of deflation of saline-filled implant due to interference by a ring of fibrous tissue from the capsule over the seal valve. (Horiz Med 2015; 15(1): 71-75)

Key words: Mammary Augmentation, Mammary Deflation, Capsule. (Source: MeSH NLM). 


\section{INTRODUCCIÓN}

El aumento mamario es uno de los procedimientos quirúrgicos estéticos más realizados dentro de la práctica del Cirujano Plástico durante más de 50 años (1).

El primer implante mamario fue introducido por Cronin y Brauer en 1962, relleno de gel de silicon y fabricado por la corporación Dow Corning (2).

Posteriormente, se desarrolló el primer implante relleno de solución salina, que fue introducido por Arion, de Francia, en 1965 (3).

Desde entonces, ha existido un incremento estable de la cirugía de aumento de mamas debido a la importancia del desarrollo de la imagen corporal, cambios de expectativas sociales, y la aceptación creciente de cirugía estética en los Estados Unidos y el mundo.

En el 2012, se realizaron, sólo en Estados Unidos; 330,631 operaciones de implante mamario, siendo éste, el procedimiento quirúrgico estético más frecuente en mujeres de dicho país (4).

Las causas de deflación de los implantes rellenos de solución salina, hasta hoy, permanecen obscuras. La deflación, es una complicación sabida, con una incidencia reportada entre el 2 y el 76 \% (5-7).

Estudios multicéntricos recientes, han demostrado que los índices de deflación, se encuentran entre el 5.5 y $8.3 \%(8,9)$.

La deflación espontánea cuenta con aproximadamente $75 \%$ de estos casos (9). En la mayoría, es indoloro y ocurre dentro de las primeras 24 horas y, es menos común, luego de unos días o semanas.

Si la deflación ocurre, el paciente puede incomodarse, pero no representa ningún problema médico peligroso (10).

Factores de riesgo (8-11)

- el tamaño,

- mayores de $450 \mathrm{cc}$,
- posición del implante,

- el llenado por debaj o o encima del indicado,

- colocación intraluminal de antibióticos,

- corticoides intraluminales y

- colocar antibióticos en el bolsillo.

\section{CASO CLÍNICO}

Reportamos un caso muy inusual de deflación de un implante de solución salina,debido a la interferencia con la válvula, por un anillo fibroso, fenómeno reportado también por Slavin (11).

Mujer de 33 años, con aumento mamario bilateral, con implantes de Solución salina Mentor Siltex, de 300 cc, operada en Octubre del 2003.(Fig. 1).

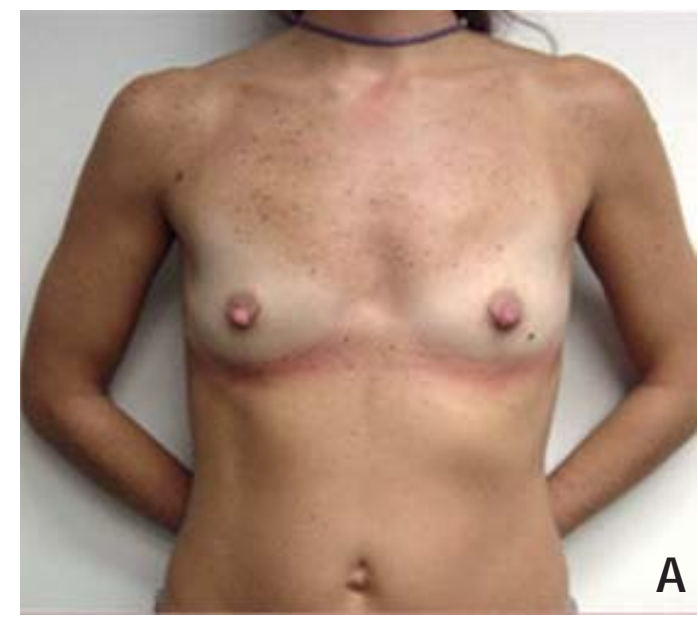

Figura 1. A Pre- Qx

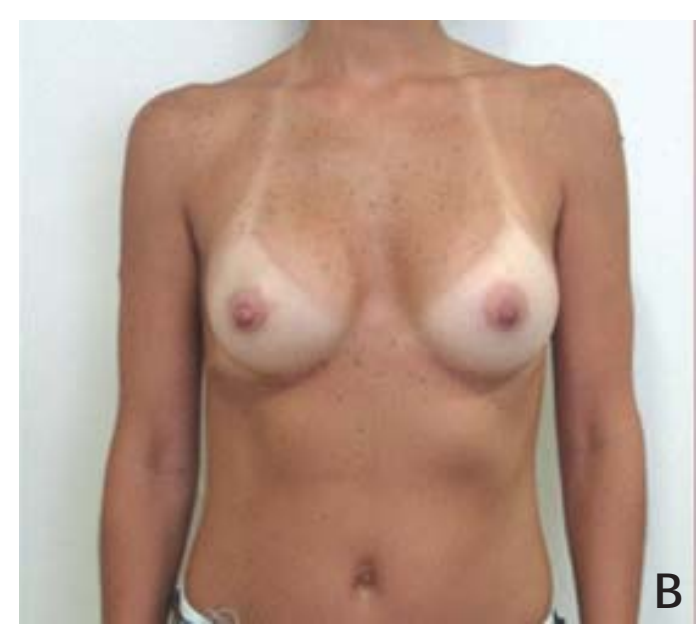

Figura 1.B Post Qx: 2 años, 6 meses 
Con controles satisfactorios al mes, 2 meses, 6 meses, al año, 2 años y 4 años.

En Octubre de 2009, (6 años después), regresa a la consulta, refiriendo una tumoración en mama izquierda. La que se confirma por mamografía: formación nodular (3-6 mm), de tipo sólida, peri areolar en la Mama izquierda, ubicada en el cuadrante súpero externo, en el radio de las $2 \mathrm{pm}$, a $1 \mathrm{~cm}$. del pezón, de contornos bien delimitados con eco interna homogénea de apariencia benigna.

Concluyendo el estudio de control a los 6 meses.
Un segundo estudio, por ecografía, determinó: focalización de mama izquierda, que sugiere quiste de contenido denso y festonamiento de borde anterior de prótesis mamaria izquierda.

Frente a estos 2 estudios, se decide observación y control a los 6 meses.

Cinco meses después (6 Años, 6 meses), acude con deflación del implante izquierdo. (Fig. 2).
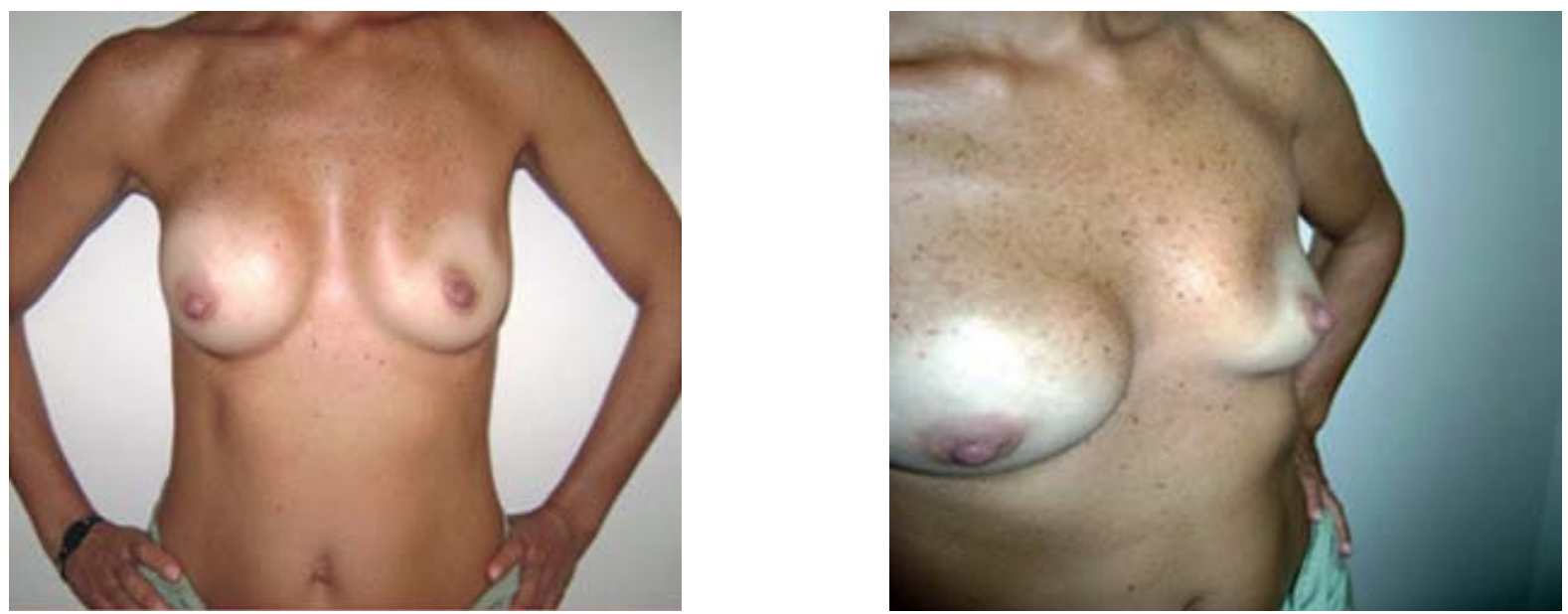

Figura 2. Frente y perfil

Se decide, la explantación y cambio de implantes esta vez por unos de mayor volumen y relleno de gel silicon con cobertura lisa. (Fig. 3).

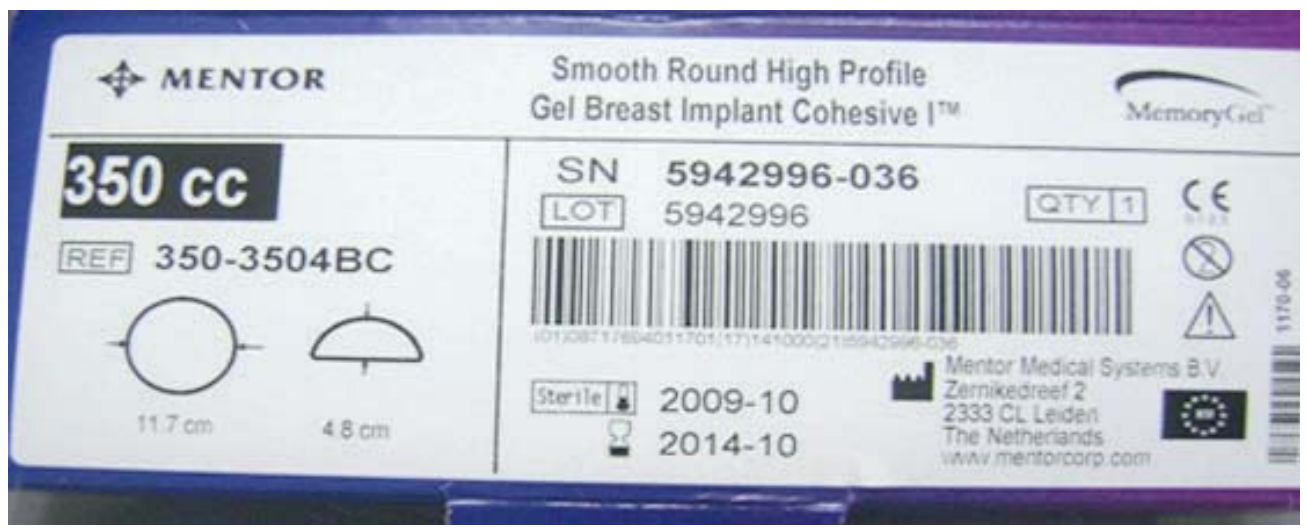

Figura 3.- Implante MENTOR de Gel de Silicon de 350 cc liso 
En el acto operatorio, se halló un anillo fibroso muy característico, que rodeaba y se conectaba a la banda del enchufe de sellado, jalándola por fuera de su posición normal. (Fig. 4).

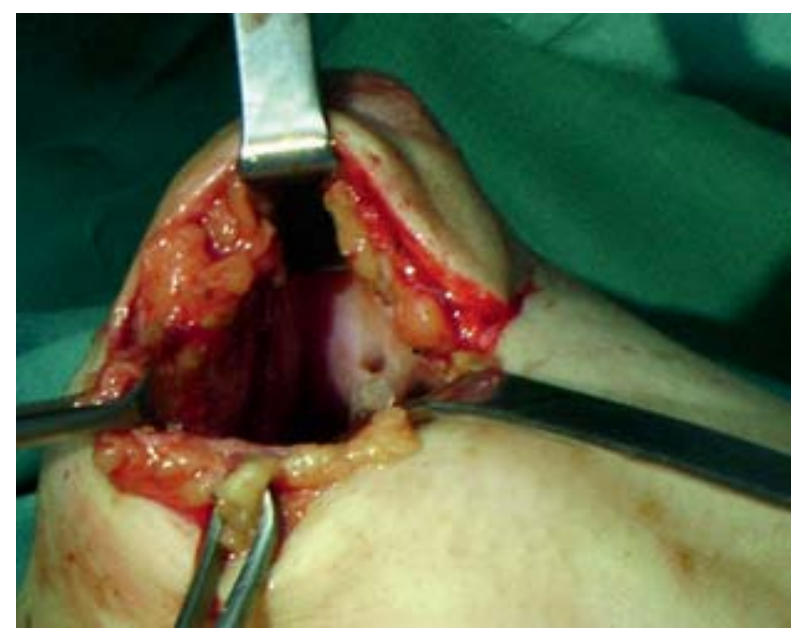

Figura 4. - Anillo Fibroso: Ring Pulls Plug

No se encontró otro sitio de fuga que sugiriera la pérdida.

Sugerimos, que los persistentes movimientos de tracción de este anillo, podrían haber interferido con el enchufe y mecanismo de la válvula, lo que llevó a la deflación, tal como lo expresó Slavin.

Figura 5

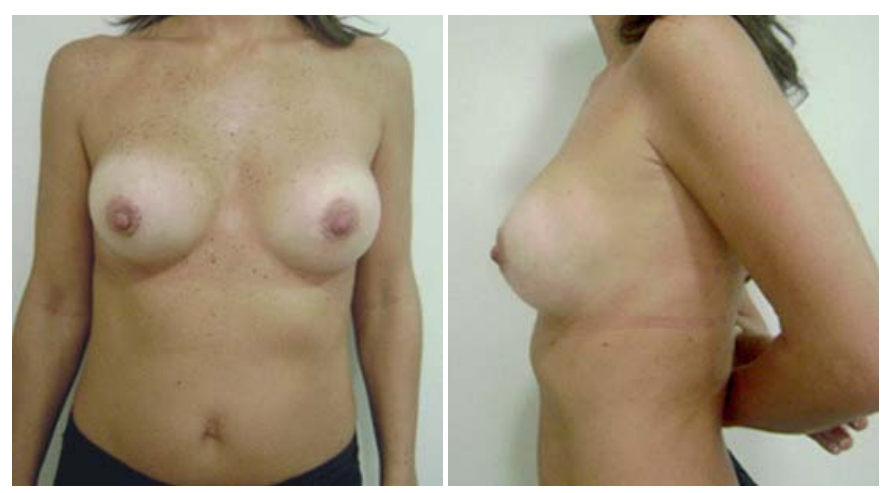

Figura 5. Post Qx 15 días: Frente y perfil

\section{DISCUSIÓN}

Para entender el porqué, se decidió en esta oportunidad por el implante relleno de gel de silicon, tendríamos que remontarnos y conocer la historia y evolución de los implantes mamarios.

Hoy se sabe que los implantes de solución salina tienen una pérdida de volumen de $1 \%$ por año (12). Frente a esta pérdida,también de gel de silicon, a principios de 1992, entre crecientes preocupaciones, controversias y procesos judiciales contra fabricantes de implantes rellenos de gel de silicon; la FDA (Administración de Drogas y Alimentos estadounidense) publicó una moratoria voluntaria 0 , restricción contra el empleo de implantes rellenos de gel de silicon para el aumento estético de Mamas $(13,14)$, esperando que surjan nuevos estudios de seguridad.

Con esta restricción, los implantes de solución salina eran la única opción disponible para pacientes que buscan la cirugía de aumento de mamas. Esta restricción, duró casi 15 años y en el 2006, terminó el experimento.

Siendo aprobado por la FDA, el uso de implantes rellenos de gel para el aumento estético mamario (14) y los implantes rellenos de silicona de las marcas: Allergan y Mentor, de venta en el mercado Americano (15-17).

La moratoria voluntaria, sirvió como una práctica de diligencia, llevando a cabo estudios y una gestión eficiente y de cuidado, para brindar seguridad a los pacientes, logrando la aprobación de la comunidad médica, el gobierno, y, lo más importante: la de los pacientes mismos.

Desde entonces, las reacciones han sido positivas, la Sociedad Americana de Estadística de Cirugía Plástica Nacional, muestra que un número creciente de mujeres opta por el aumento de mamas cada año.

De 212,500 procedimientos realizados en el 2000 , creció a 291,350 en el 2005, con un aumento del $37 \%$ en 5 años (18). Y a 6 años, marcó un 
aumento del $55 \%$. Volviendo a ser el procedimiento quirúrgico estético más frecuente y popular desde la moratoria de 1992 (19). Una razón importante para la popularidad de esta operación, ha sido probablemente, el aumento de la autoestima en relación a la imagen corporal, proporcionado por el aumento de mamas (20). En conclusión, en este reporte observamos que la interposición de un anillo fibroso en la válvula de sellado sería una rara causa de deflación mamaria.

\section{REFERENCIAS BIBLIOGRÁFICAS}

1. Adams W, Jr. The Process of Breast Augmentation: Four Sequential Steps for Optimizing Outcomes for Patients. Plastic Reconstr Surg2008:122(6): 1892 -1900.

2. Cronin TD, Brauer RO. Augmentation mammaplasty. Surg Clin North Am 1971;51:441-452.

3. Maxwell GP, Gabriel A. The evolution of breast implants. Clin Plast Surg 2009;36:1-13.

4. Procedural statistics. Available at: http://www.surgery.org/ media/statistics. 2012. Accessed January, 2014.

5. Lavine D.M. Saline inflayable prostheses: 14 years experience. Aesthetic Plast Surg 1993; 17: 325.

6. Mladic R.A. "No-touch"Submuscular saline breast augmentation technique. Aesthetic Plastic Surgery 1993; 17: 183.

7. Gylbert L, Asplund O, Jurell G. Capsular contracture after breast reconstruction with silicone-gel and saline-filled implants: A 6-year follow-up. Plastic \&Reconstructive Surg 1990; 85: 373.

8. Gutowski KA, Mesna GT, Cunningham BI. Saline-filled breast implants: A Plastic Surgery Educational Foundation multicenter outcome study. Plastic \&Reconstructive Surgery 1997; 100: 1019.

9. Cunningham BL, Lokeh A, Gutowski KA. Saline-filled breast implant safety and efficacy: A multicenter retrospective review. Plastic \& Reconstructive Surgery 2000; 105:2143.

10. Rheingold L, Yoo R, CourtissE. Experience with 326 Inflatable Breast Implants. Plastic \& Reconstructive Surgery 1994; 93: 118.

11. Slavin SA. Why saline breast implants deflate. Presented at the 65th Annual meeting of the American Society of Plastic and Reconstructive Surgeons. Dallas Texas, 1996.

12. Stevens WG, Hirsch EM, Stoker DA, Cohen R. "A Comparison of 500 Prefilled Textured Saline Breast Implants versus 500 Standard Textured Saline Breast Implants: Is There a Difference in Deflation Rates? “. Plastic and Reconstructive Surgery, Vol 117(7), 2175-78, June 2006. History of breast implants.

\section{Fuente de financiamiento}

Este estudio ha sido autofinanciado por el autor.

\section{Conflictos de interés}

El autor declara no tener ningún conflicto de interés
13. Rohrich R. Silicone Breast Implants: Outcomes and Safety UpdatePlastic \& Reconstructive Surgery 2007; 120(7):1S-3S.

14. American Society of Plastic Surgeons. Plastic Surgery Societies applaud the FDA's decision to approve silicone breast implants (press release), November 17, 2006. Available at: http://www. plasticsurgery.org/media/press_releases/Approve-SiliconeBreast-Implants.cfm .Accessed June 19, 2007.

15. Tillman D. Department of Health and Human Services, Food and Drug Administration. Letter to Mentor Corporation, Department of Clinical and Regulatory Affairs re: Silicone Gel-Filled Breast Implants, November 17, 2006.

16. Silicone gel-filled breast implants approved. F.D.A. Consum 2007;41: 8.

17. American Society of Plastic Surgeons. 2000/2004/2005 national plastic surgery statistics. Available at:

http:// www. plasticsurgery.org/ public_education/loader. cfm?url_/commonspot/security/getfile.cfm\&PagelD_17870 .Accessed July 8, 2007.

18. American Society of Plastic Surgeons. 2006 quick facts: Cosmeticand reconstructive plastic surgery trends. Available at: http://www.plasticsurgery.org/media/statistics/loader.cfm?url/ commonspot/security/getfile.cfm\&PagelD_23625. Accessed June19, 2007.

19. Figueroa-Haas CL. Effect of breast augmentation mammoplastyon self-esteem and sexuality: A quantitative analysis. Plast. Surg. Nurs 2007;27: 16.

\section{Correspondencia:}

Guillermo M. Wiegering Cecchi

Dirección: Clinica Javier Prado.

Av. Javier Prado Este 499 Of. 215. San Isidro Perú. Teléfono: +(51 1) 999263360 / Of: 221-3314 Correo electrónico: gwiegering@gmail.com 